\title{
Efficient energy transfer between nanocrystalline YAG:Ce and TRITC
}

\author{
Sander F. Wuister, * Celso de Mello Donegá and Andries Meijerink \\ Debye Institute, Condensed Matter and Interfaces, Utrecht University, P.O. Box 80 000, 3508 \\ TA, Utrecht, The Netherlands. E-mail: S.F.Wuister@phys.uu.nl; Fax: +31 30 2532403; \\ Tel: +31302532207
}

Received 27th January 2004, Accepted 27th February 2004

First published as an Advance Article on the web 4th March 2004

\begin{abstract}
Luminescent nanocrystalline (NC) $\mathrm{Ce}^{3+}$-doped yttrium aluminum garnet $\left(\mathrm{Y}_{3} \mathrm{Al}_{5} \mathrm{O}_{12}: \mathrm{Ce}-\mathrm{YAG}: \mathrm{Ce}\right)$ is prepared by a combustion synthesis technique that yields a highly porous three-dimensional network of interconnected $18 \mathrm{~nm}$ nanocrystals. Tetramethyl rhodamine isothiocyanate (TRITC) was conjugated to NC YAG:Ce by using glycine as a bridging molecule. Efficient energy transfer is observed from the $\mathrm{Ce}^{3+}$ ions to the TRITC molecules upon photoexcitation of the NC YAG: Ce-TRITC conjugates.
\end{abstract}

Resonant energy transfer (ET) between a donor in the excited state and a neighboring acceptor is commonly observed between dyes, ${ }^{1}$ quantum dots and dyes, ${ }^{2,3}$ quantum dots, ${ }^{4,5}$ and metal ions. ${ }^{6}$ The ET process can occur via several mechanisms, but the electric dipole-electric dipole interaction is the most effective one over relatively long distances (up to several tens of angstroms, with a $r^{-6}$ distance dependence). ${ }^{6}$ This mechanism is particularly efficient when both donor and acceptor have allowed electric dipole resonant transitions.

ET between suitable probes can be used to obtain structural information or monitor dynamic processes in biological systems but its usefulness is severely limited by fast photobleaching when dyes are used for tagging the target molecules. Semiconductor quantum dots are more stable than dyes ${ }^{7}$ but still suffer from photoinduced degradation processes. Luminescent ions in inorganic phosphors offer the prospect of a greatly superior photo-stability associated with high quantum yields. Intermittence in emission (blinking), which is a problem in certain applications of single quantum dots, is not expected for inorganic phosphor nanocrystals as they contain a large number of luminescing ions (typically between 10 and 10000 depending on the size of the nanocrystal and the concentration of luminescing ions). In the past few years there has been a growing interest in the properties of nanocrystalline lanthanide-doped insulating phosphors, and several well-known materials, such as $\mathrm{Y}_{2} \mathrm{O}_{3}: \mathrm{Eu}^{3+},{ }^{3-10} \mathrm{Lu}_{2} \mathrm{O}_{3}: \mathrm{Eu}^{3+},{ }^{31} \mathrm{YVO}_{4}$ : $\mathrm{Eu}^{3+},{ }^{12} \mathrm{LaPO}_{4}: \mathrm{Ln}^{3+}\left(\mathrm{Ln}=\mathrm{Eu}\right.$ or $\left.\mathrm{Ce} / \mathrm{Tb}^{13}\right), \mathrm{YbPO}_{4}: \mathrm{Er}^{3+},{ }^{34}$ and $\mathrm{Y}_{3} \mathrm{Al}_{5} \mathrm{O}_{12}: \mathrm{Ln}^{3+} \mathrm{Ln}=\mathrm{Nd}, \mathrm{Eu}, \mathrm{Tb}, \mathrm{Tm}$ or $\mathrm{Ce}^{15-19}$ ), have been prepared in nanocrystalline form. The application of lanthanide-doped nanocrystalline phosphors as biological labels would however strongly limit the photon turnover rates due to the long ( $\mu$ s to $\mathrm{ms}$ ) decay time of their intraconfigurational $4 \mathrm{f}^{n}$ luminescence. One of the few exceptions for visible-emitting phosphors is $\mathrm{Y}_{3} \mathrm{Al}_{5} \mathrm{O}_{12}$ :Ce (YAG:Ce). In YAG:Ce the 5d state of $\mathrm{Ce}^{3+}$ is situated at a low energy resulting in a fast intraconfigurational $5 \mathrm{~d} \rightarrow 4 \mathrm{f}$ luminescence in the visible. The luminescence life time of this emission is $65 \mathrm{~ns}^{20}$ This luminescence decay time is ideally suited for time-gated detection of biological species, since it is slower than the autofluorescence background decay common to many biological species (typically a few ns), but is still fast enough to ensure a high turnover rate. Additional attractive features are the excitation in the blue (which further decreases the autofluorescence background obtained under UV excitation) and the large Stokes shift of the luminescence. The strong electric dipole of the $\mathrm{Ce}^{3+} \mathrm{f}-\mathrm{d}$ emission also makes NC YAG:Ce a promising candidate as donor for efficient ET processes to dyes.

In order to evaluate the potentiality of $\mathrm{NC}$ YAG:Ce as a probe in biological systems the ET from NC YAG:Ce to tetramethyl rhodamine isothiocyanate (TRITC) was investigated. TRITC is chemically conjugated to highly luminescent NC YAG:Ce by using glycine as a linker molecule. Steady state (excitation and emission spectra), time-domain (fluorescence decay times) measurements and photobleaching experiments clearly demonstrate the occurrence of ET from $\mathrm{Ce}^{3+}$ to TRITC molecules in the NC YAG:Ce-TRITC conjugates.

Nanocrystalline YAG:Ce $(0.5 \mathrm{~mol} \%)$ was prepared by using a combustion synthesis technique similar to that reported in, ${ }^{10}$ but optimized to yield nanoparticles smaller than $20 \mathrm{~nm}$ with a small degree of sintering. Glycine was used as a fuel and the metal nitrates as oxidizers. Typically, $3 \mathrm{mmol}$ of $\mathrm{Ln}\left(\mathrm{NO}_{3}\right)_{3} \cdot 4 \mathrm{H}_{2} \mathrm{O}(\mathrm{Ln}=\mathrm{Y}$ and $\mathrm{Ce}), 5 \mathrm{mmol}$ of $\mathrm{Al}\left(\mathrm{NO}_{3}\right)_{3} \cdot 9 \mathrm{H}_{2} \mathrm{O}$ and $14 \mathrm{mmol}$ of glycine were dissolved in $4 \mathrm{~mL}$ of demiwater and the resultant solution was dried overnight at $80^{\circ} \mathrm{C}$. The resulting transparent gel was placed into a preheated furnace at $650^{\circ} \mathrm{C}$ for $30 \mathrm{~min}$ in air. The as-synthesized product was a foamy porous gray body, which was shown by $\mathrm{X}$-ray powder diffraction to be amorphous. This product was subsequently treated at $1150^{\circ} \mathrm{C}$ for $90 \mathrm{~s}$ under air, yielding a highly porous yellow body. Fig. 1 shows the X-ray powder diffraction patterns of the final product and of a microcrystalline

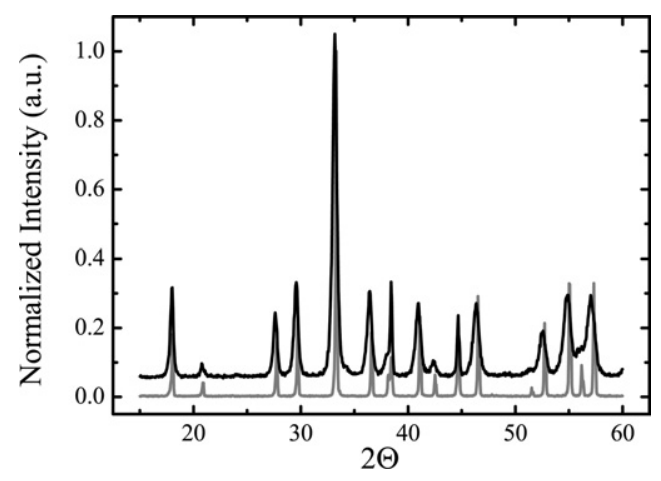

Fig. 1 X-ray powder diffraction patterns $(\mathrm{Cu} \mathrm{K} \alpha)$ of NC YAG:Ce (black) and microcrystalline YAG:Ce (dark gray). 
powder sample of YAG:Ce (commercial phosphor purchased from Nichia Co.). It can be concluded that the final product consists of single phase crystalline YAG. The peak broadening and the small shift of the peak maxima to lower angles can be ascribed to a finite size effect, indicating that the sample consists of nanocrystalline domains. The mean size of these domains, determined from the full width at half maximum of the peak with the Debye-Scherrer formula ${ }^{21}$ is $18 \mathrm{~nm}$. Thermal treatments at temperatures higher than $1150^{\circ} \mathrm{C}$ and/or for longer times yield larger average nanocrystal sizes and more pronounced sintering. Temperatures below $1000^{\circ} \mathrm{C}$ yield amorphous white samples (non-luminescent). The use of other fuels such as urea or citric acid leads to slightly larger YAG:Ce nanocrystals $(23 \mathrm{~nm})$, lower quantum yields and a larger degree of sintering.

The NC YAG:Ce samples are highly friable and can be easily ground to a powder and broken apart into NC clusters $(100-1000 \mathrm{~nm})$ by sonic waves (sonic bath). The transmission electron microscopy (TEM) image of one such NC YAG:Ce cluster is depicted in Fig. 2A, which clearly shows that the NC YAG:Ce samples consist of a highly porous and interconnected three-dimensional network of nanoparticles. The high degree of crystallinity of these YAG:Ce nanoparticles is confirmed by both high-resolution TEM (Fig. 2B) and selected area electron diffraction (inset of Fig. 2A). The size of the YAG:Ce nanocrystals obtained from TEM and HR-TEM is consistent with the average size determined from XRD $(\sim 20 \mathrm{~nm})$.

To conjugate TRITC to NC YAG:Ce a portion of $35 \mathrm{mg}$ NC YAG:Ce (a slightly yellow powder) in $10 \mathrm{~mL}$ ultra-pure water was mixed thoroughly with $35 \mathrm{mg}$ glycine (aminoacetic acid) and left overnight. Excess of glycine was removed by centrifuging and several times rinsing with ultra pure water. Glycine was chosen as the linker molecule because it can bind to both TRITC and NC YAG:Ce. The carboxylic group of glycine is expected to strongly bind to the surface of the YAG:Ce nanocrystals via the $\mathrm{Y}^{3+}$ and $\mathrm{Al}^{3+}$ ions, whereas its primary amine group reacts with the isothiocyanate group of TRITC. The use of TRITC as functionalised dye allows for selective excitation of the YAG:Ce at $440 \mathrm{~nm}$ since the absorption of TRITC at this wavelength is very low. A disadvantage of TRITC as a dye is the spectral overlap between the emission of YAG:Ce and TRITC which complicates a quantitative analysis of the energy transfer efficiency from the emission spectra. To the glycine capped NC YAG:Ce $35 \mu \mathrm{g}$ of TRITC in $10 \mathrm{~mL}$ methanol was added and the sample was left overnight. The excess of TRITC was removed by centrifuging and rinsing twice with methanol. The sample (hereafter named

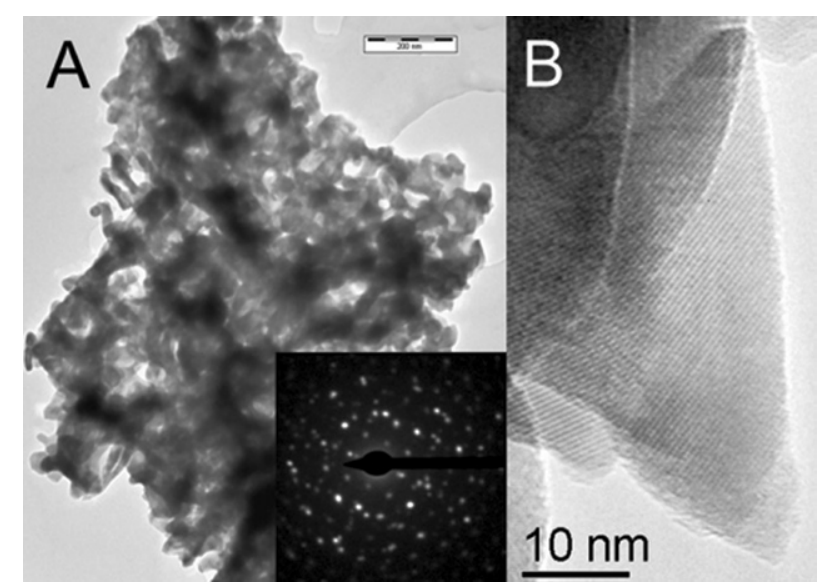

Fig. 2 (A) Transmission electron micrograph of NC YAG:Ce. A selected area electron diffraction pattern is presented in the inset. (B) shows a HR-TEM image of a selected region in the same sample of NC YAG:Ce.
TRITC-NC YAG:Ce conjugate) is a light pink powder and was dried in air prior to characterization. It should be mentioned that treatment of YAG:Ce with glycine and Rhodamine B (a dye molecule similar to TRITC, but without the isothiocyanate group) did not lead to a dye-NC YAG:Ce conjugate, showing that the presence of an isothiocyanate group is crucial for the formation of the conjugate.

Steady-state emission and excitation spectra were recorded with a SPEX Fluorolog spectrofluorometer, model F2002, equipped with two monochromators (double-grating, $0.22 \mathrm{~m}$, SPEX 1680) and a $450 \mathrm{~W}$ xenon lamp as the excitation source. Luminescence decay curves were measured using a Pico Quant PDL 800 -B laser $\left(\lambda_{\mathrm{ex}}=440 \mathrm{~nm}, 2.5 \mathrm{MHz}\right.$ repetition rate, $55 \mathrm{ps}$ pulse width) as an excitation source. The luminescence was dispersed by a monochromator $(0.1 \mathrm{~m}$ focal length, 1350 line $\mathrm{mm}^{-1}$ grating, blazed at $500 \mathrm{~nm}$ ) and detected by a fast Hamamatsu H5738P-01 photomultiplier tube. The decay curves were obtained by time correlated single photon counting (TCPCS) via time-to-amplitude conversion (TAC) with a Time Harp 100 computer card. The ratio of stop to start pulses was kept low (below 0.05 ) to assure good statistics.

Fig. 3 shows the excitation spectra $\left(\mathrm{A}, \lambda_{\mathrm{em}}=580 \mathrm{~nm}\right)$ and emission spectra $\left(B, \lambda_{\mathrm{ex}}=440 \mathrm{~nm}\right)$ of tetramethyl rhodamine isothiocyanate (TRITC) in water (dark gray curves), NC YAG: Ce (light gray curves) and TRITC-NC YAG:Ce conjugate (black curves). The emission spectrum of NC YAG:Ce $(0.5 \mathrm{~mol} \%)$ consists of a broad band with maximum around $550 \mathrm{~nm}$ that is ascribed to a $5 \mathrm{~d} \rightarrow 4 \mathrm{f}$ transition. The $4 \mathrm{f} \rightarrow 5 \mathrm{~d}$ excitation transitions yield broad bands at around $260 \mathrm{~nm}$ (not shown), $340 \mathrm{~nm}$ and $450 \mathrm{~nm}$. The band at $260 \mathrm{~nm}$ is very weak because the upper $5 \mathrm{~d}$ states of $\mathrm{Ce}^{3+}$ in YAG have energies within the conduction band of the host and therefore excitation in these levels results mainly in quenching. ${ }^{20} \mathrm{The}^{\mathrm{Ce}^{3+}}$ $5 \mathrm{~d}$ level populated by the transition occurring at $340 \mathrm{~nm}$ is just below the YAG conduction band and will also be quenched to some extent at room temperature. ${ }^{20}$ There are no significant differences between the emission and excitation spectra of NC YAG:Ce and bulk YAG:Ce, as expected considering that the band-gap of YAG is very large $(7 \mathrm{eV})$ and that the electronic transitions involved in the luminescence are localized on the $\mathrm{Ce}^{3+}$ ion. Quantum size effects, as observed for the luminescence of nanocrystalline semiconductors, are not expected for the luminescence of $\mathrm{Ce}^{3+}$ in NC YAG. The quantum efficiency of the NC YAG:Ce sample is lower than that of a bulk sample $\left(\sim 50 \%\right.$ at $\lambda_{\text {ex }}=430 \mathrm{~nm}$, instead of the $70 \%$ observed for commercial Nichia phosphor). The lower quantum efficiency of the nanocrystalline samples can be ascribed to a decrease in the excitation efficiency due to light scattering and to quenching and competitive absorption by surface defects (e.g. oxygen vacancies and/or disorder at grain boundaries ${ }^{20}$ ). The quantum efficiencies can be improved by longer thermal treatments or higher temperatures, which however

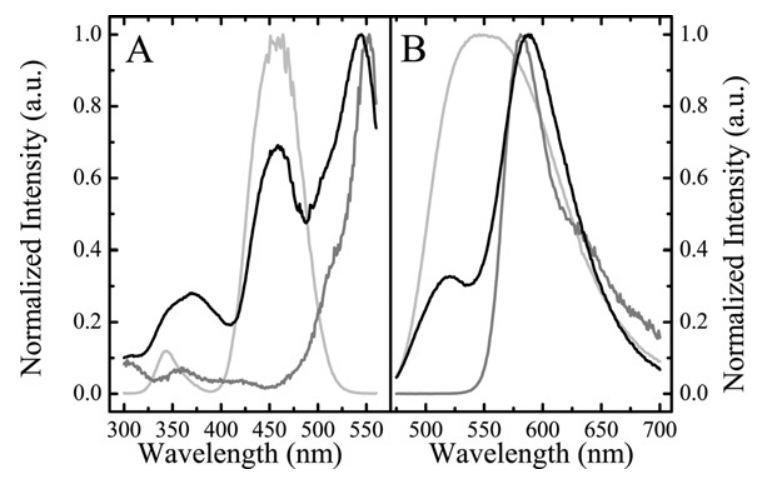

Fig. 3 (A) Normalized excitation spectra $\left(\lambda_{\mathrm{em}}=580 \mathrm{~nm}\right)$ and (B) emission spectra $\left(\lambda_{\text {ex }}=440 \mathrm{~nm}\right)$ of NC YAG:Ce (light gray), TRITC (dark gray), and YAG:Ce-TRITC conjugate (black). 
lead to undesired nanocrystal growth and sintering. The luminescence quantum efficiency is given by the number of emitted photons divided by the number of absorbed photons, and was determined by comparison with standard phosphors, following the method of de Mello Donega et al. ${ }^{22}$ This method is accurate within $10 \%$.

It is clear from the excitation spectra in Fig. 3A that selective excitation of NC YAG:Ce is possible at $440 \mathrm{~nm}$ (TRITC hardly absorbs at this wavelength). The black curve in Fig. $3 \mathrm{~B}$ shows the emission spectrum of TRITC-NC YAG:Ce conjugate under $440 \mathrm{~nm}$ excitation. The weak band around 520 $\mathrm{nm}$ can be ascribed to $\mathrm{Ce}^{3+}$ emission, while the peak around $580 \mathrm{~nm}$ is due to TRITC emission. This provides a clear indication that ET from $\mathrm{Ce}^{3+}$ ions to TRITC takes place, since the TRITC emission dominates the emission spectrum despite its negligible absorption at $440 \mathrm{~nm}$, whereas the strong absorption of YAG:Ce at $440 \mathrm{~nm}$ yields only a weak $\mathrm{Ce}^{3+}$ luminescence. We would like to point out that the spectral overlap between the emission of the donor and the absorption of the acceptor, needed for ET via dipole-dipole interaction, is good since the maximum of the donor $\left(\mathrm{Ce}^{3+}\right)$ emission and the acceptor (TRITC) absorption band are both situated around $550 \mathrm{~nm}$. In the excitation spectrum of TRITC-NC YAG:Ce conjugate (Fig. 3A, black curve) the peak at $\sim 550 \mathrm{~nm}$ is due to direct excitation of the TRITC. The peak around $450 \mathrm{~nm}$ is ascribed to direct excitation of $\mathrm{Ce}^{3+}$ ions. It is noteworthy that the relative intensity of the peak around $375 \mathrm{~nm}$ for the TRITC-NC YAG:Ce conjugate is larger than expected by a simple convolution of the excitation bands of NC YAG:Ce and TRITC, suggesting an increased excitation efficiency due to resonance between the $\mathrm{Ce}^{3+} 5 \mathrm{~d}$ level at $3.64 \mathrm{eV}(340 \mathrm{~nm})$ and TRITC excited states. This resonance would lead to efficient ET between the two levels, competing with the depopulation of the $\mathrm{Ce}^{3+} 5 \mathrm{~d}$ level via the YAG conduction band.

The observation of $\mathrm{Ce}^{3+}$ excitation bands in the excitation spectrum of the TRITC emission at $580 \mathrm{~nm}$ in the conjugate sample provides further evidence for the occurrence of $\mathrm{Ce}^{3+} \rightarrow$ TRITC ET, but it is not unambiguous since the broad emission band of $\mathrm{Ce}^{3+}$ overlaps with the emission of TRITC. The luminescence decay curve of the $\mathrm{Ce}^{3+}$ emission provides a more direct way to probe whether $\mathrm{Ce}^{3+} \rightarrow$ TRITC ET is indeed taking place. Fig. 4 shows the luminescence decay curves of NC YAG:Ce (in light gray) and of TRITC-NC YAG:Ce conjugate (in black). The decay curves are recorded monitoring luminescence at $520 \mathrm{~nm}$ to avoid detection of the TRITC emission. The decay curve of the $\mathrm{Ce}^{3+}$ ions in YAG is nearly monoexponential and has a decay time of $60 \mathrm{~ns}$. This is very close to the reported decay time of $\mathrm{Ce}^{3+}$ ions in bulk YAG sample $\left(65 \mathrm{~ns},{ }^{20}\right)$. This is surprising since the effective refractive index is lower in NC YAG:Ce than in the bulk material due to the presence of a lower refractive index material

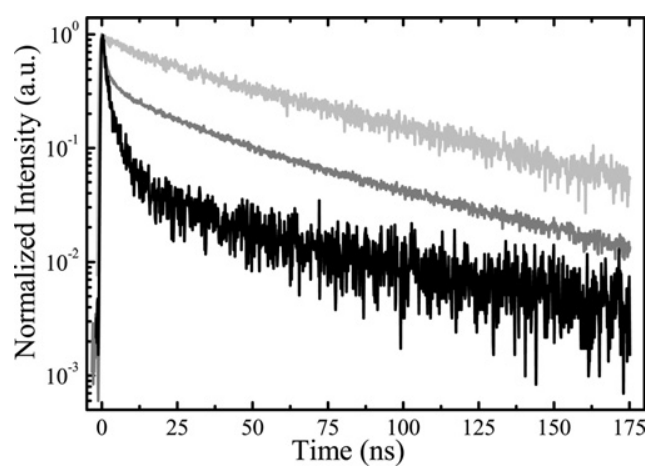

Fig. 4 Luminescence decay curves $\left(\lambda_{\mathrm{ex}}=440 \mathrm{~nm}, \lambda_{\mathrm{em}}=520 \mathrm{~nm}\right)$ of NC YAG:Ce (light gray) and YAG:Ce-TRITC conjugate (black). The decay curve of YAG:Ce-TRITC conjugate after photobleaching is shown in dark gray. (liquid helium or air) around the nanocrystals and within a distance of the order of the wavelength of light. It is expected that, through local field effects, the lower effective refractive index would reduce the radiative decay rate of the $\mathrm{Ce}^{3+}$ emission in NC YAG:Ce in comparison with the bulk. This effect has already been observed for $\mathrm{NC}_{2} \mathrm{O}_{3}: \mathrm{Eu}^{3+} .{ }^{23}$ In the case of NC YAG:Ce, however, the filling fraction is not known, what makes the comparison with the previous studies on $\mathrm{NC}$ $\mathrm{Y}_{2} \mathrm{O}_{3}: \mathrm{Eu}^{3+}$ difficult. Further, non-radiative processes are more important for the nanocrystal than for the bulk materials due to the larger surface to volume ratio, which is reflected in an initial deviation from single-exponential decay and a lower quantum efficiency. The slightly faster initial decay, observed for NC YAG:Ce can explain why the luminescence decay time measured, defined as the time at which the emission intensity has dropped to $1 / \mathrm{e}$ of its initial value, is shorter for the NC YAG:Ce despite the lower effective refractive index. For the TRITC-NC YAG:Ce conjugate (black curve) a fast initial decay in the luminescence intensity of the $\mathrm{Ce}^{3+}$ ion is observed, indicating the presence of an additional decay channel for the $\mathrm{Ce}^{3+}$ ions in the conjugate, which can be ascribed to resonant ET from the $5 \mathrm{~d}$ excited states of $\mathrm{Ce}^{3+}$ in YAG to acceptor excited states of TRITC molecules at the nanocrystals surface. Radiative transfer from the $\mathrm{Ce}^{3+}$ ions to the TRITC molecules may also occur, but would not lead to shorter life times for the donor states. The fact that coupling to TRITC results in significantly faster luminescence decay curve shows that non-radiative energy transfer via dipole-dipole interaction is dominant. The average ET rate can be estimated from the decay curves and is $\sim 10^{8}-10^{9} \mathrm{~s}^{-1}$. Since both the $\mathrm{Ce}^{3+}$ and the TRITC emissions are allowed electric-dipole transitions, the dipole-dipole interaction will be strong allowing efficient ET to occur if the $\mathrm{Ce}^{3+}-$ TRITC distances are sufficiently small. Using the Förster-Dexter theory for energy transfer via dipole-dipole interaction an estimate of the energy transfer rate can be made. The critical distance for energy transfer (where the radiative rate equals the energy transfer rate) is given by ${ }^{24}$

$$
R_{\mathrm{c}}^{6}=3 \times 10^{12} f_{\mathrm{A}} E^{-4} \mathrm{SO}
$$

Here $f_{\mathrm{A}}$ is the oscillator strength of the acceptor transition, $E$ is the energy at the maximum of spectral overlap (in eV) and SO is the spectral overlap integral. The oscillator strength as determined from the TRITC absorption spectrum is about 0.5 . The spectral overlap determined from the YAG:Ce emission and TRITC absorption spectrum is $2 \mathrm{eV}^{-1}$. This gives a critical distance for energy transfer from $\mathrm{Ce}^{3+}$ to TRITC of about $7 \mathrm{~nm}$. As a result, energy transfer rates of $10^{8} \mathrm{~s}^{-1}$ and faster are expected for $\mathrm{Ce}^{3+}$ ions in an outer shell of $5 \mathrm{~nm}$ thick in the NC. This is quite consistent with the observations. The luminescence decay curves show that a majority of the $\mathrm{Ce}^{3+}$ show a fast decay due to efficient energy transfer to TRITC. For the average size of $18 \mathrm{~nm}$ YAG:Ce nanocrystals more than $90 \%$ of the $\mathrm{Ce}^{3+}$ ions will be within a $5 \mathrm{~nm}$ distance from the surface and will transfer the excitation energy with transfer rates of $10^{8}$ $\mathrm{s}^{-1}$ and higher to TRITC molecules at the surface. It should be noted that a $18 \mathrm{~nm}$ diameter YAG:Ce $(0.5 \mathrm{~mol} \%)$ nanocrystal contains about $1700 \mathrm{Ce}^{3+}$ ions statistically distributed over all available $\mathrm{Y}^{3+}$ sites. This will cause a distribution in the TRITC to $\mathrm{Ce}^{3+}$ distances. Since ET by electric dipole-electric dipole interaction scales with $\mathrm{r}^{-6}, \mathrm{Ce}^{3+}$ ions closer to the surface will transfer their energy much more efficiently then ions in the core of the nanocrystal. This distribution of donor-acceptor distances will result in a distribution of ET rates between $\mathrm{Ce}^{3+}$ ions and TRITC molecules. The size polydispersity inherent to the NC YAG:Ce samples further broadens the distribution of ET rates. Therefore only an average ET rate can be determined from the experimental data. ET is not only possible between the $\mathrm{Ce}^{3+}$ ions and the TRITC molecules but also between $\mathrm{Ce}^{3+}$ ions. Therefore $\mathrm{Ce}^{3+}$ ions deep inside the 


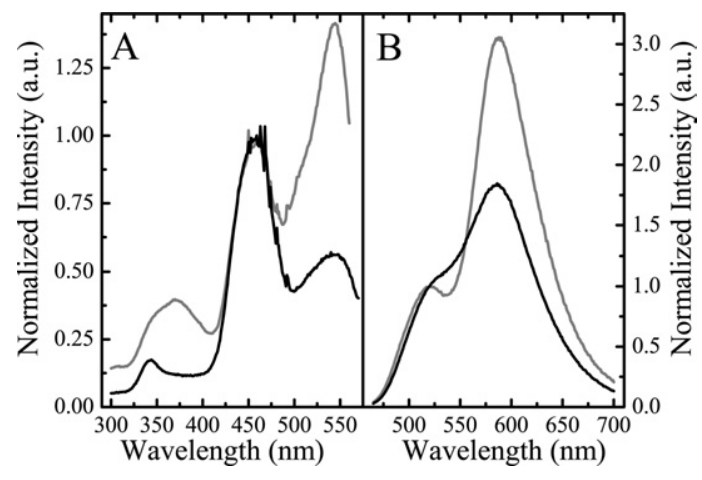

Fig. 5 (A) Excitation spectra $\left(\lambda_{\mathrm{em}}=580 \mathrm{~nm}\right)$ and (B) emission spectra $\left(\lambda_{\mathrm{ex}}=440 \mathrm{~nm}\right)$ of YAG:Ce-TRITC conjugate before (dark gray) and after (black) $65 \mathrm{~h}$ excitation with $300 \mathrm{~nm}$ from a $450 \mathrm{~W}$ Xenon lamp. Both emission and excitation spectra were normalized to the $\mathrm{Ce}^{3+}$ peak.

nanocrystals may also transfer their energy to $\mathrm{Ce}^{3+}$ ions closer to the surface which subsequently transfer their excitation energy to the TRITC molecules, thus increasing the ET rate. A more quantitative analysis of the energy transfer process is not possible in view of the various uncertainties discussed above.

Photobleaching can be used as a control experiment ${ }^{25}$ to show that the decay time shortening of the $\mathrm{Ce}^{3+}$ luminescence observed in the TRITC-YAG:Ce conjugate is indeed due to energy transfer. TRITC will degrade after being exposed to UV light for a long period, while YAG:Ce is photostable. Fig. 5 shows the excitation (A) and emission (B) spectra of the TRITC-YAG:Ce conjugate prior to (dark gray) and after (black) 65 hours of irradiation with $300 \mathrm{~nm}$ light from a $450 \mathrm{~W}$ xenon lamp. The color of the sample after irradiation changed from pink to slight yellow, clearly indicating that the photodecomposition of the TRITC molecules has occurred. In the excitation spectrum of the photobleached sample (Fig. 5A) the peak around $550 \mathrm{~nm}$ (due to direct excitation of TRITC) is strongly reduced, which implies that the amount of TRITC in the sample has decreased. However, not all the TRITC molecules were bleached, despite the long period of UV radiation, possibly because the strong scattering characteristic of nanoporous materials limits the penetration depth of the light.

In the emission spectra (Fig. 5B) a strong decrease in the emission intensity of the peak around $580 \mathrm{~nm}$ (the TRITC emission) is observed after bleaching. Since less TRITC is present at the surface, the average distance between $\mathrm{Ce}^{3+}$ ions and TRITC molecules increases. Therefore the likelihood of $\mathrm{Ce}^{3+}$ to TRITC energy transfer is diminished and the fraction of radiative recombination of the $\mathrm{Ce}^{3+}$ ions increases. Both contributions will be reflected in the decay curves of the photobleached TRITC-YAG:Ce conjugate sample (Fig. 4, dark gray curve). The fast initial decay is still present, but has a much smaller contribution than prior to the photobleaching, indicating that a smaller population of $\mathrm{Ce}^{3+}$ ions have a TRITC molecule at distances short enough to allow efficient ET to take place. This is also reflected in the larger contribution of the exponential decay tail due to the radiative decay of the $\mathrm{Ce}^{3+}$ ions without neighboring TRITC molecules.

In conclusion, we have demonstrated that efficient energy transfer (ET rates of $\sim 10^{8}-10^{9} \mathrm{~s}^{-1}$ ) from $\mathrm{Ce}^{3+}$ ions to TRITC molecules occurs in the TRITC-NC YAG:Ce conjugate. Also, we have shown that nanocrystalline YAG:Ce can be successfully conjugated to organic molecules, therefore establishing its potentiality as a biolabel. Additional experiments aiming at imaging and spectrally resolving single YAG:Ce nanocrystals have also been successfully carried out and will be reported elsewhere.

\section{Acknowledgements}

This work was financially supported by Utrecht University within the 'Breedte-strategie' program 'Physics of Colloidal Matter'. The authors are grateful to Dr Hans Meeldijk (EMSADebye Institute, Utrecht University, The Netherlands) for TEM and HRTEM measurements.

\section{References}

1 T. Förster, Ann. Phys. VI, 1948, 2, 55.

2 C. E. Finlayson, D. S. Ginger and N. C. Greenham, Chem. Phys. Lett., 2001, 338, 83.

3 O. Schmelz, A. Mews, T. Basche, A. Herrmann and K. Muellen, Langmuir, 2001, 17, 2861.

4 C. R. Kagan, C. B. Murray, M. Nirmal and M. G. Bawendi, Phys. Rev. Lett., 1996, 76, 1517.

5 S. A. Crooker, J. A. Hollingsworth, S. Tretiak and V. I. Klimov, Phys. Rev. Let., 2002, 89, 186802.

6 B. Henderson and G. F. Imbusch, Optical Spectroscopy of Inorganic Solids, Oxford Science Publications, Oxford, 1989, ch. 10.

7 M. Bruchez Jr., M. Moronne, P. Gin, S. A. Weiss and A. P. Alivisatos, Science, 1998, 281, 2013.

8 B. M. Tissue, Chem. Mater., 1998, 10, 2837.

9 R. Schmechel, M. Kennedy, H. von Seggern, H. Winkler, M. Kolbe, R. A. Fischer, L. Xaomao, A. Benker, M. Winterer and H. Hahn, J. Appl. Phys., 2001, 89, 1679.

10 R. S. Meltzer, S. P. Feofilov, B. M. Tissue and H. B. Yuan, Phys. Rev. B, 1999, 60, R14012.

11 E. Zych, A. Meijerink and C. de Mello Donegá, J. Phys.: Condens. Matter, 2003, 15, 5145.

12 K. Riwotzki and M. Haase, J. Phys. Chem. B, 2001, 105, 12709.

13 K. Riwotzki, H. Meyssamy, H. Schnablegger, A. Kornowski and M. Haase, Angew. Chem., Int. Ed. Engl., 2001, 40, 573.

14 O. Lehmann, H. Meyssamy, K. Kömpe, H. Schnablegger and M. Haase, J. Phys. Chem. B, 2003, 107, 7449.

15 M. Veith, S. Mathur, A. Kareiva, M. Jilavi, M. Zimmer and V. Huch, J. Mater. Chem., 1999, 9, 3069.

16 H. Yukiya, T. Haganuma, K. Sue, T. Adschiri and K. Arai, Mat. Res. Bull., 2003, 38, 1257.

17 O. A. Lopez, J. McKittrick and L. E. Shea, J. Lumin., 1997, 71, 1 .

18 D. Hreniak and W. Strek, J. Alloys Compd., 2002, 341, 183.

19 Chung-Hsin Lu and R. Jagannathan, Appl. Phys. Lett., 2002, 80, 3609 .

20 E. Zych, C. Brecher and J. Glodo, J. Phys: Condens. Matter., 2000, 12, 1947.

21 B. D. Cullity, Elements of X-ray Diffraction, Addison-Wesley, Massachusetts, 1978.

22 C. De Mello Donega, S. J. L. Ribeiro, R. R. Gonçalves and G. Blasse, J. Phys. Chem. Solids, 1996, 57, 1727.

23 R. S. Meltzer, S. P. Feofilov, B. Tissue and H. B. Yuan, Phys. Rev $B, 1999,60,14012$.

24 G. Blasse and B. C. Grabmaier, Luminescent Materials, SpringerVerlag, Berlin, 1994, p. 93.

25 U. Kubitscheck, M. Kircheis, R. Schweitzer-Stenner, W. Dreybrodt, T. Jovin and I. Pecht, Biophys. J., 1991, 60, 307. 